\title{
Sustained coevolution of phage Lambda and Escherichia coli involves inner- as well as outer-membrane defences and counter-defences
}

\author{
Alita R. Burmeister ${ }^{1,2, *}, \dagger$, Rachel M. Sullivan ${ }^{1,2} \neq$, Jenna Gallie $3,4,5 \S$ and Richard E. Lenski $i^{1,2}$
}

\begin{abstract}
Bacteria often evolve resistance to phage through the loss or modification of cell surface receptors. In Escherichia coli and phage $\lambda$, such resistance can catalyze a coevolutionary arms race focused on host and phage structures that interact at the outer membrane. Here, we analyse another facet of this arms race involving interactions at the inner membrane, whereby E. coli evolves mutations in mannose permease-encoding genes manY and manZ that impair $\lambda$ 's ability to eject its DNA into the cytoplasm. We show that these man mutants arose concurrently with the arms race at the outer membrane. We tested the hypothesis that $\lambda$ evolved an additional counter-defence that allowed them to infect bacteria with deleted man genes. The deletions severely impaired the ancestral $\lambda$, but some evolved phage grew well on the deletion mutants, indicating that they regained infectivity by evolving the ability to infect hosts independently of the mannose permease. This coevolutionary arms race fulfils the model of an inverse gene-for-gene infection network. Taken together, the interactions at both the outer and inner membranes reveal that coevolutionary arms races can be richer and more complex than is often appreciated.
\end{abstract}

\section{INTRODUCTION}

An issue of longstanding interest is whether the coevolution of bacteria and virulent (lytic) phages involves endless rounds of bacterial defences and phage counter-defences. Based on experiments in chemostats, Lenski and Levin [1] suggested that bacteria typically had the upper hand, as Escherichia coli often eventually evolved resistance by deleting or inactivating the phage's specific receptor, which the phage could not readily overcome. This resistance did not imply the extinction of the phage, however, because it often reduced the bacteria's competitiveness for resources. Instead, the typical outcome was coexistence of resistant and sensitive bacteria, with the latter more efficient at exploiting resources and thus able to sustain the phage's persistence $[2,3]$. A study of cyanobacteria and their phages in the marine environment also supported this pattern [4].
On the other hand, Lenski and Levin also pointed out that bacteria would lose the upper hand if the phage targeted a receptor that was essential for the bacteria to survive in their current environment. They cited then-recent work by Williams Smith and Huggins [5, 6], who showed that they could successfully treat mice with otherwise lethal bacterial infections using a phage that specifically targeted a receptor required for the bacteria to colonize the mice. As the problem of bacterial resistance to antibiotics has grown, similar strategies are now being tested in which phage that specifically target drug efflux pumps are deployed as therapeutic agents [7-9]. In the meantime, yet other forms of bacteria-phage coevolution have been discovered, including CRISPR systems in bacteria and countermeasures to avoid these defences in phage [10-13].

Another part of the argument that bacteria had the upper hand in the coevolutionary arms race depended on the idea that, while phages could often counter minor mutations in

Received 29 April 2021; Accepted 04 May 2021; Published 25 May 2021

Author affiliations: 'Department of Microbiology and Molecular Genetics, Michigan State University, East Lansing, MI, USA; ${ }^{2}$ BEACON Center for the Study of Evolution in Action, Michigan State University, East Lansing, MI, USA; ${ }^{3}$ Department of Biology, University of Washington, Seattle, WA, USA; ${ }^{4}$ Department of Environmental Microbiology, Eawag, Dübendorf, Switzerland; ${ }^{5}$ Department of Environmental Systems Science, ETH Zürich, Zürich, Switzerland.

*Correspondence: Alita R. Burmeister, alita.burmeister@yale.edu

Abbreviations: GFG, gene-for-gene; IGFG, inverse gene-for-gene.

†Present address: Department of Ecology and Evolutionary Biology, Yale University, New Haven, CT, USA

‡Present address: Waisman Center, University of Wisconsin, Madison, WI, USA

§Present address: Department of Evolutionary Theory, Max Planck Institute for Evolutionary Biology, 24306 Plön, Germany.

Supplementary material is available with the online version of this article.

001063 (c) 2021 The Authors 
receptors, it was much more difficult for them to evolve the ability to use another receptor if the bacteria simply stopped producing the usual receptor [1]. However, more recent work has shown that some host-phage pairs can undergo longer coevolutionary cycles involving defences and counter-defences at the outer membrane [14-16], and some phages can evolve to use new receptors even on a short time scale [17]. This coevolutionary dynamic - in which hosts lose structures exploited by specific pathogens, and those pathogens evolve to exploit alternative structures - is called inverse gene-for-gene (IGFG) coevolution [18-21]. This IGFG framework is useful for representing changes in coevolving communities of bacteria and phage (Fig. 1). For example, if phage cannot evolve to exploit new features after bacteria have evolved resistance, then phage populations may be evolutionarily static $[22,23]$. Conversely, if phage exploit essential features of the bacteria that cannot be eliminated, then the host's evolution is constrained and phage infectivity may remain elevated $[6,8]$. Our study builds on one such example of IGFG coevolution, in which it was discovered that populations of a virulent strain of phage $\lambda$ often evolved the ability to use another outer-membrane receptor after coevolving E. coli reduced their expression of the receptor that the phage had initially exploited $[17,24]$.

Phage $\lambda$ requires a two-step infection process to cross the outer and inner bacterial membranes (Fig. 1). The $\lambda$ tail initiates infection at the outer membrane of the cell, where its J protein fibres adsorb to the bacterial protein LamB $[25,26]$. The tail proteins $\mathrm{V}$ and $\mathrm{H}$ allow $\lambda$ to enter the periplasm and thereby interact with the mannose permease proteins (encoded by $\operatorname{man} Y$ and $\operatorname{man} Z$ ) in the inner membrane, which $\lambda$ uses to eject its genome into the cytoplasm [27-30]. Resistance to $\lambda$ can occur by blocking $\lambda$ 's entry at either the outer or inner membrane, with resistance mutations typically mapping to $\operatorname{lam} B$, lamB's positive regulator malT $[25,26]$, or the mannose permease genes $[27,28,30]$ (Fig. 1). It has been shown that sensitive E. coli and lytic $\lambda$ can coexist, along with resistant $E$. coli mutants, in both continuous [31] and batch culture regimes [17]. Previous analysis of this coevolving system has revealed IGFG dynamics focused on outer-membrane defences and counter-defences. That is, E. coli often first evolves malT mutations that reduce LamB expression, resulting in increased resistance to $\lambda[17,31,32]$, and $\lambda$ then regains infectivity through mutations in the $J$ gene that increase its adsorption rate and fitness $[31,33]$. In some, but not all, experiments, specific sets of $J$ mutations allow the novel exploitation of a second outer-membrane protein, OmpF, catalyzing further evolution including mutations in the ompF gene [17, 34].

Despite extensive knowledge about the evolution of the initial (adsorption) and final (lysis) steps of $\lambda$ infection of $E$. coli, much less is known about the evolution of the genetic networks during other stages of infection, including $\lambda$ 's passage through the periplasmic space and the ejection of its DNA into the host cytoplasm. Meyer et al. [17] found that $E$. coli coevolving with $\lambda$ often acquired mutations that affected their ability to grow on mannose, which

\section{Impact Statement}

Laboratory studies of coevolution help us understand how host defences and pathogen counter-defences change over time, which is often essential for predicting the future dynamics of host-pathogen interactions. One particular model, termed inverse gene-for-gene coevolution, predicts that coevolution proceeds through alternating steps, whereby hosts lose the features exploited by pathogens, and pathogens evolve to exploit alternative features. Using a classic model system in molecular biology, we describe the nature and timing of a previously overlooked step in the coevolution of E. coli and bacteriophage lambda. Our work demonstrates that this mode of coevolution can profoundly reshape the interactions between bacteria and phage.

presumably were favoured because they disrupt entry of the phage genome via the mannose permease. In this study, we examine how this coevolutionary arms race - previously focused on the cell's outer membrane - also set off an arms race involving the host's inner membrane, including the mechanism $\lambda$ uses to eject its DNA through that membrane and into the bacteria's cytoplasm.

\section{METHODS}

\section{Bacteria and phage strains}

Meyer et al. [17] founded 96 replicate cultures with E. coli B strain REL606 and lytic phage $\lambda$ cI26, serially passaged the communities for 20 days, and froze mixed-community samples daily. Some of the phage populations evolved the ability to use the outer-membrane protein $\mathrm{OmpF}$ as a receptor, some of the bacterial populations evolved mutations that affected mannose metabolism and some communities changed in both respects. We obtained phage isolates from two of the populations (Table 1, Pop-A and Pop-B) that changed in both of these key respects; in each case, however, the isolates were taken 4 days before the phage had evolved the new ability to use the OmpF receptor (Table 1, Supplementary Results and Discussion). E. coli K12 strains BW25113, JW1807 and JW1808 are from the Keio collection [35]. REL606 $\triangle m a n Z$ was constructed using a two-step allelic exchange (Supplemenatry Methods, Tables S1 and S2).

\section{Phage growth assays}

We measured the population growth of the ancestral and evolved phages under the same culture conditions as those in which the communities evolved (Supplementary Methods) [17]. The initial densities were $\sim 9 \times 10^{6}$ cells $\mathrm{ml}^{-1}$ and $\sim 1 \times 10^{4}$ phage $\mathrm{ml}^{-1}$. We calculated the phage's net population growth as the ratio of its final density after 1 day to its initial density; we show the resulting net growth on a $\log _{10}$-transformed scale. We enumerated the initial and final phage populations using dilution plating and soft agar 
(a)

\section{Gene-for-gene coevolution:}

Host evolves by gaining resistance genes, and pathogen evolves by losing genes that elicit host defence.

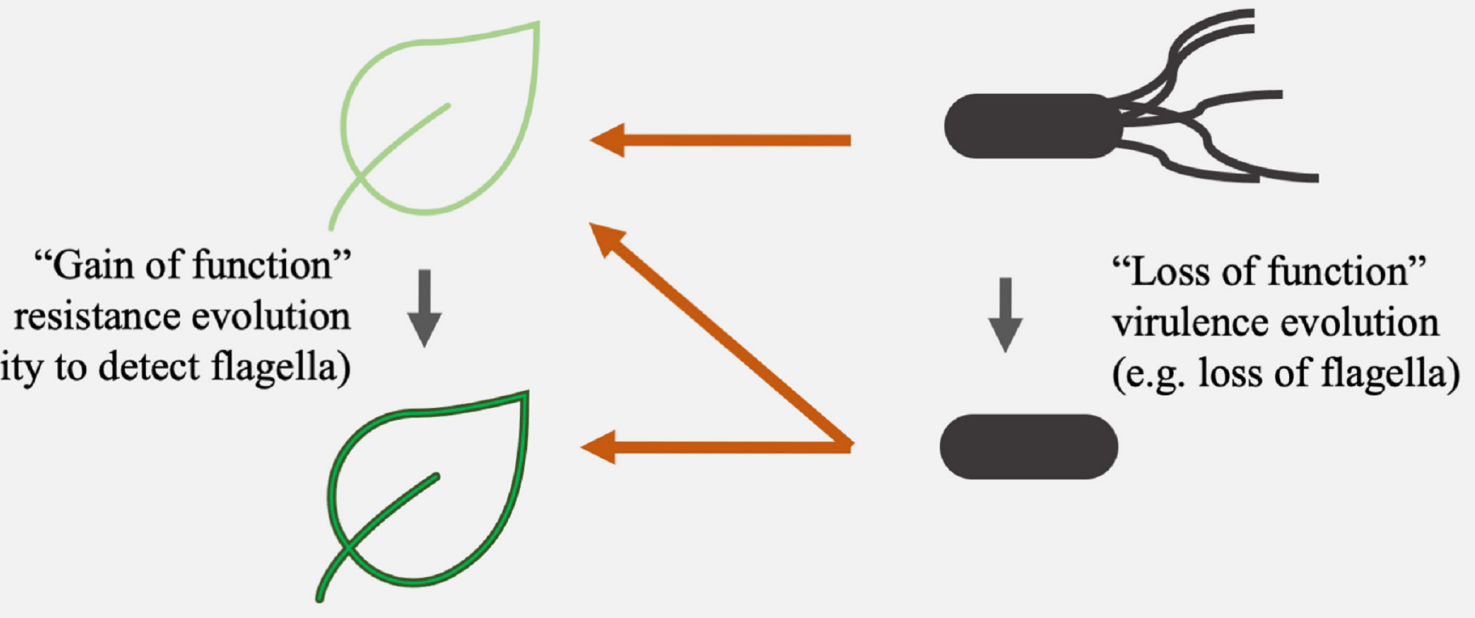

(b)

Inverse gene-for-gene coevolution:

Host evolves resistance by losing structures, and pathogen evolves to exploit other structures.

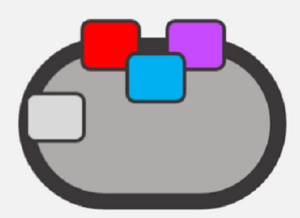

"Loss of function"

(e.g. loss of LamB and maltose transport)

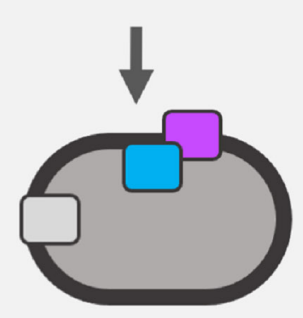

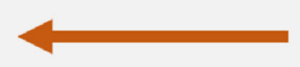

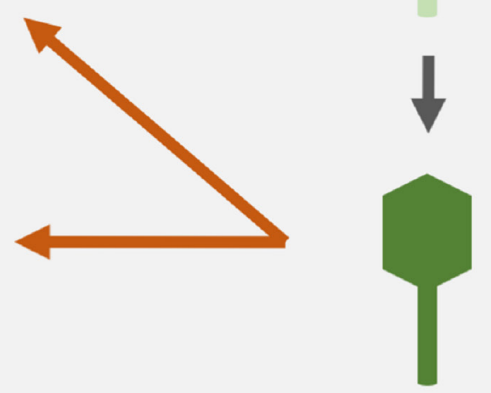

"Gain of function" (e.g. ability to infect through OmpF)

Fig. 1. Genetic interaction networks during gene-for-gene (GFG) coevolution (a) and inverse gene-for-gene (IGFG) coevolution (b). In both scenarios, host alleles affect selection on pathogen phenotypes, and pathogen alleles influence selection on host phenotypes. However, the two models have different implications for understanding historical coevolution and predicting future changes. During GFG coevolution, hosts evolve resistance by gaining resistance genes, and pathogens evolve by losing genes that elicit host defences. GFG coevolution is common among plants and their bacterial pathogens; it may also occur in bacteria-phage interactions that involve restriction-modification and CRISPR defences. During IGFG coevolution, pathogen infectivity requires the exploitation of specific host features, and resistance involves eliminating the exploited features. Unlike in the GFG model, host defences in the IGFG model do not require pathogen recognition, and the pathogen's evasion of host resistance does not require the loss of a defence elicitor. 
Table 1. E. coli and phage $\lambda$ strains used in this study

\begin{tabular}{|c|c|c|}
\hline Strain & Description & Relevant characteristics \\
\hline \multicolumn{3}{|l|}{ Bacteria Clones: } \\
\hline REL606 & E. coli B ancestor of coevolution experiment & $m a l T^{+}, o m p F^{+}, \operatorname{man} Y^{+}, \operatorname{manZ}^{+}$ \\
\hline REL606 $\Delta$ man $Z$ & $\operatorname{man} Z$ deletion derived from REL606 ${ }^{*}$ & $\Delta m a n Z$ \\
\hline BW25113 & E. coli K12 parental strain of Keio collection & $m a l T^{+}, o m p F^{+}, \operatorname{man} Y^{+}, \operatorname{manZ}^{+}$ \\
\hline JW1808 & man $Z$ deletion in Kei o collection & $\Delta m a n Z$ \\
\hline DH5a & Strain used for $\lambda$ plaque-based enumeration & $m a l T^{+}, o m p F^{+}, \operatorname{man} Y^{+}, \operatorname{man} Z^{+}$ \\
\hline \multicolumn{3}{|l|}{ Phage Clones: } \\
\hline cI26 & Lytic $\lambda$ ancestor of both phage populations & Requires E. coli LamB \\
\hline
\end{tabular}

${ }^{*}$ This strain also has three mutations that have no known relevance to interactions with phage $\lambda$ (Supplementary Results and Discussion). For construction methods, see Supplemenatry Methods.

†For simplicity, we have designated the source populations Pop-A and Pop-B. These correspond to population numbers D9 and G9 in the original experiment described by Meyer et al. [17].

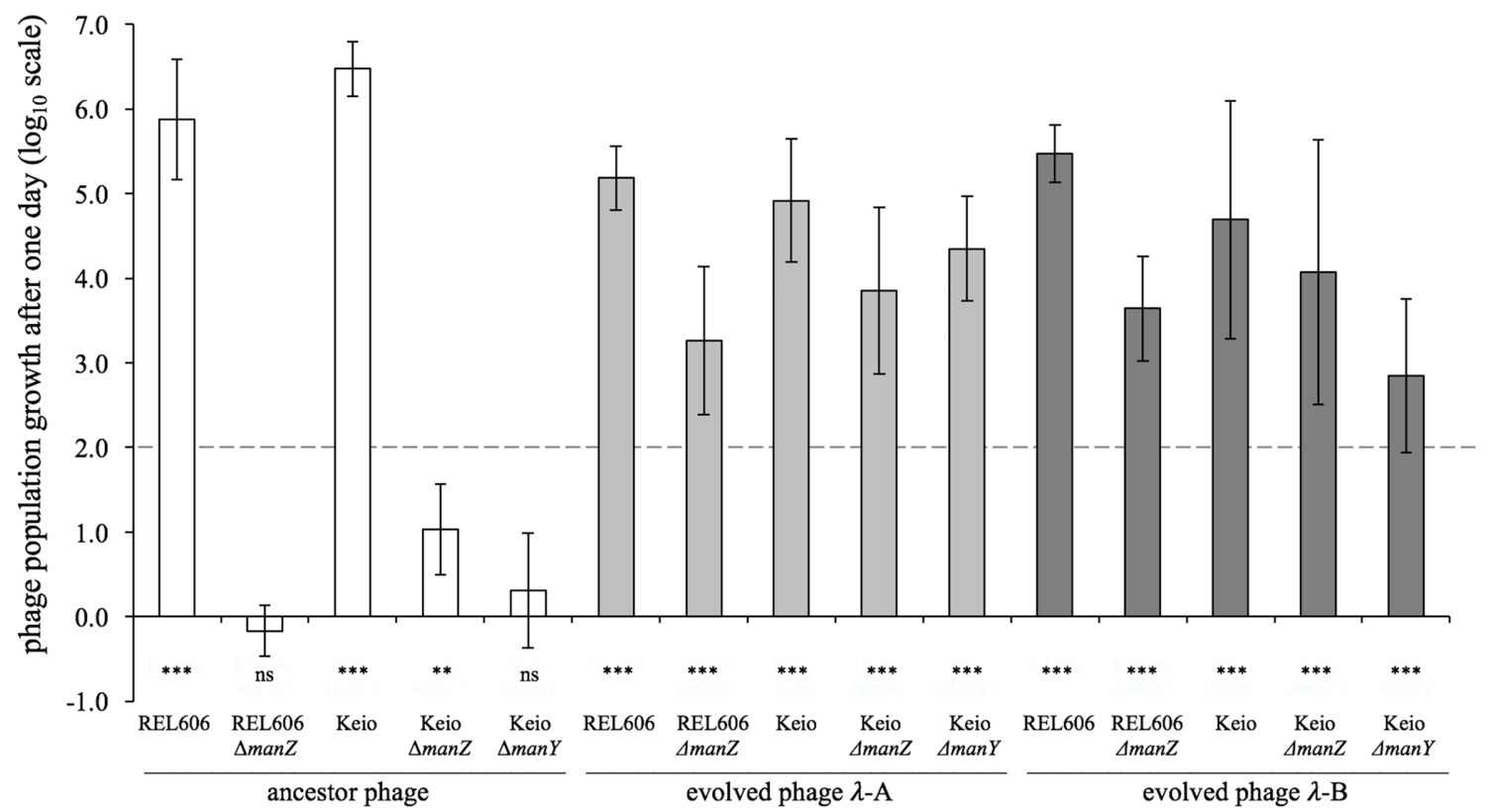

Fig. 2. Net population growth of phage $\lambda$ on wild-type, $\Delta$ manY and $\triangle$ manZ bacteria. Whether the phage could grow was assessed by performing one-tailed $t$-tests on the $\log _{10}$-transformed ratio of phage population densities at the start and end of a 1 day cycle, with the null hypothesis of zero growth $\left({ }^{* * *}, P<0.001 ;{ }^{* *}, 0.001<P<0.01\right.$; NS, not significant, $\left.P>0.05\right)$. Each test was based on five or six replicate assays. Phage isolates $\lambda$-A and $\lambda$-B evolved in a batch culture regime with 100 -fold dilution each day, and so 100 -fold growth was required for their persistence; this break-even level is indicated by the dashed line. 

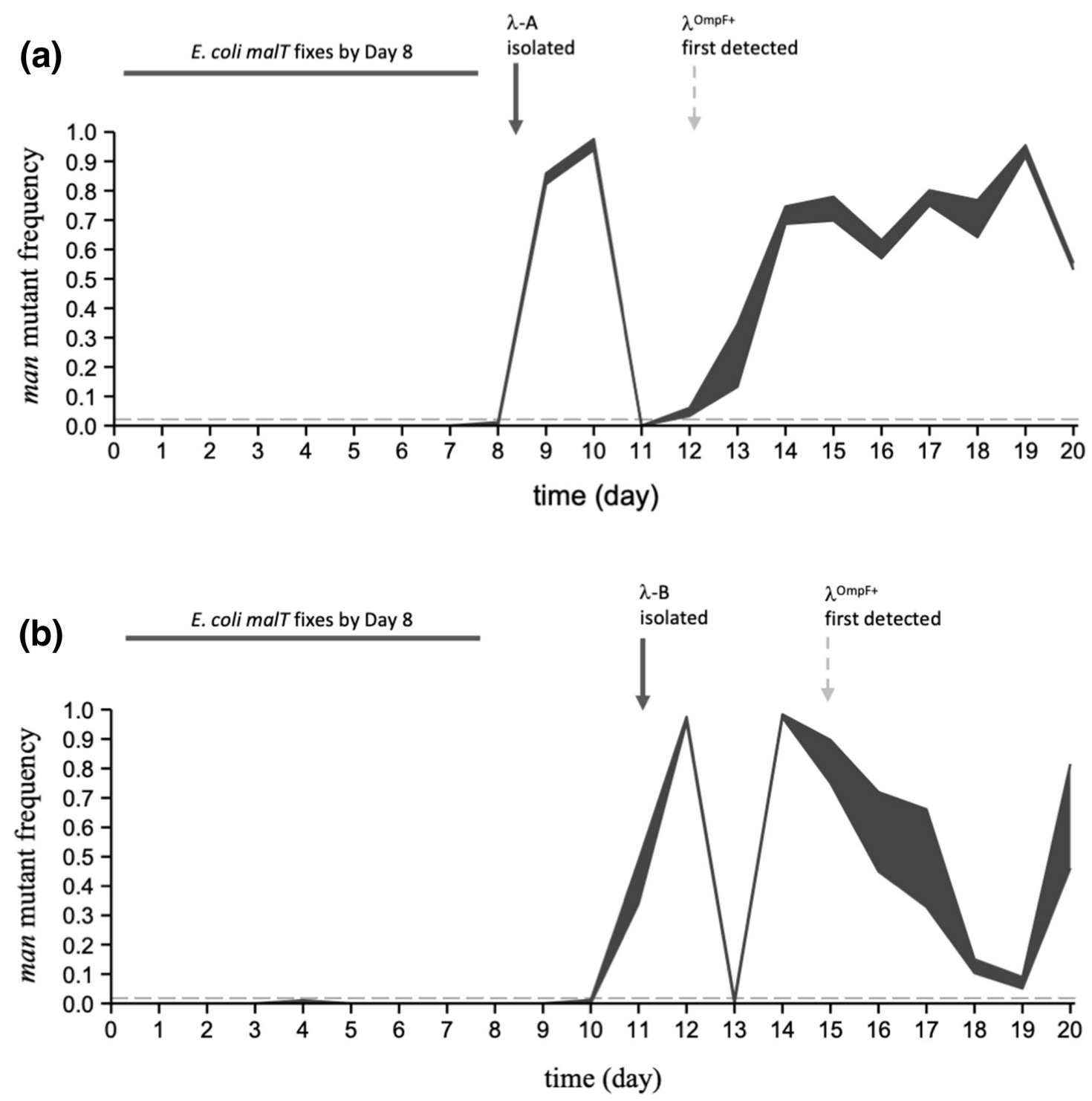

Fig. 3. Temporal dynamics of man mutants in E. coli populations Pop-A (a) and Pop-B (b). Mutant malT alleles had already reached fixation in both populations by day 8 [17]. Bacteria with man mutations, which confer resistance to the ancestral phage $\lambda$, rose to high frequencies and then declined sharply in abundance in both populations after day 8 , but before $\lambda$ had evolved to use the alternative receptor $0 \mathrm{mpF}$ (timing indicated by vertical dashed arrows). These data imply that the man mutations evolved on malT mutant backgrounds, and that $\lambda$ evolved independence of the mannose permease - causing the precipitous decline in the frequency of man mutants - before it evolved the ability to use $\mathrm{OmpF}$. The shaded regions indicate the maximum and minimum frequencies of the man mutants based on analysing two samples per population each day (mean $n=90$ colonies tested per sample, minimum 29 colonies). The horizontal grey dashed lines show the approximate limit of detection of the man mutants [0.019 for (a), 0.022 for (b)].

overlays (Supplementary Methods). We performed five or six replicate assays for each phage-host combination shown in Fig. 2.

\section{Frequency of mutants with altered mannose phenotypes}

We estimated the frequency of bacteria with mutations affecting the mannose permease by plating from the time series of frozen samples taken from populations Pop-A and
Pop-B on tetrazolium mannose agar, as done previously [17]. Mutants with reduced ability to metabolize mannose form deeply pigmented colonies that can be readily distinguished from those of the ancestral strain REL606, which forms light pink colonies on that medium.

The data are available as Supplementary Datasets S1 (net population growth of phage $\lambda$ on wild-type and knockout bacteria) and S2 (temporal dynamics of man mutants in E. coli populations). 
(a)

\title{
morphological evolution in Pop-A
}

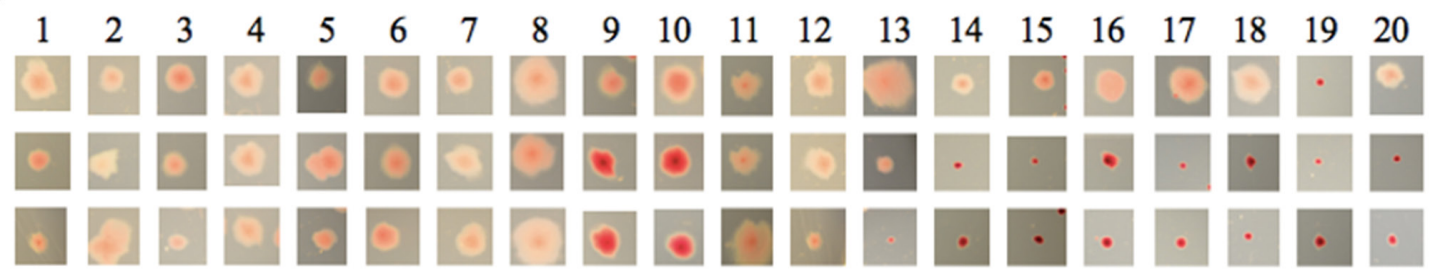

(b) morphological evolution in Pop-B

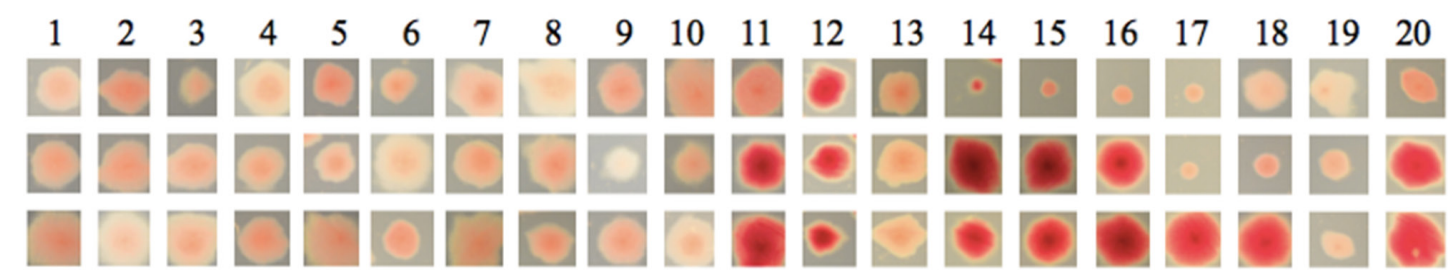

(c)

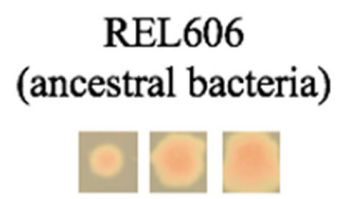

$\Delta \operatorname{manZ}$

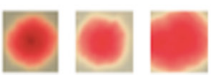

\begin{abstract}
Fig. 4. Evolution of man-related colony morphology on tetrazolium mannose agar. E. coli mutants with reduced ability to metabolize mannose form more deeply pigmented colonies than the wild-type bacteria. Three representative colonies are shown for each sample from days 1-20 of two coevolution experiments. Representative colonies within a column are from the same agar plate and shown at the same magnification after incubation for 18-21 h. (a) Pop-A. (b) Pop-B. (c) Comparison of wild-type and $\Delta m a n Z$ bacteria in the same E. coli strain B genetic background.
\end{abstract}

\section{RESULTS AND DISCUSSION}

Our experiments focus on two independently coevolved communities of mixed $E$. coli and $\lambda$ populations, designated Pop-A and Pop-B [17]. Both $\lambda$ populations evolved from a common ancestral phage (strain cI26). From each evolved population, we isolated a single phage clone: $\lambda$-A from Pop-A and $\lambda$-B from Pop-B (Table 1). Each clone was isolated 4 days before its population evolved the ability to use the OmpF receptor; hence, the phage clones were isolated on different days of the coevolution experiment performed by Meyer et al. [17].

To examine whether and how coevolution affected $\lambda$ 's dependence on the ManY and ManZ proteins, we measured the population growth of the ancestral (cI26) and the two coevolved phage isolates $(\lambda-A$ and $\lambda-B)$ on bacterial strains with and without the man $Y$ and man $Z$ genes (Table 1). Both the ancestral and evolved phage isolates grew well on bacterial strains with intact man $Y$ and man $Z$ genes, including both the ancestral E. coli B strain, REL606, used in the coevolution experiment, and the K12 genetic background in which the Keio collection was made (Fig. 2, Table 1). Deletion of either the man $Y$ or man $Z$ gene in either background severely reduced the ancestral phage's population growth. In two cases (REL606 $\triangle m a n Z$ and Keio
$\Delta$ man $Y$ ), we saw no growth whatsoever in the ancestral phage (cI26) population after $24 \mathrm{~h}$; in the other case (Keio $\Delta$ manZ), the ancestral phage population increased $\sim 10$-fold, but that was 5 orders of magnitude less than the increase on the same background with both mannose permease genes present. In striking contrast, both evolved phage isolates showed substantial growth on all three bacterial strains that lacked either the man $Y$ or man $Z$ gene (Fig. 2). These results thus indicate an inverse gene-for-gene coevolutionary interaction at the inner membrane. That is, the bacteria modified or lost the mannose permease, which the ancestral phage used to eject its genome into the cytoplasm, and the phage countered by evolving independence of that function.

To determine when the mutant mannose permease mutants arose in the two E. coli populations studied here, we plated frozen samples from the coevolution experiments on tetrazolium mannose agar, on which man mutants form pigmented colonies distinguishable from the wild-type (Supplementary Results and Discussion) [36]. We are particularly interested in the timing of the appearance of the man mutants relative to two other steps in the coevolutionary arms race that were previously characterized: (i) the malT mutations that reduced 


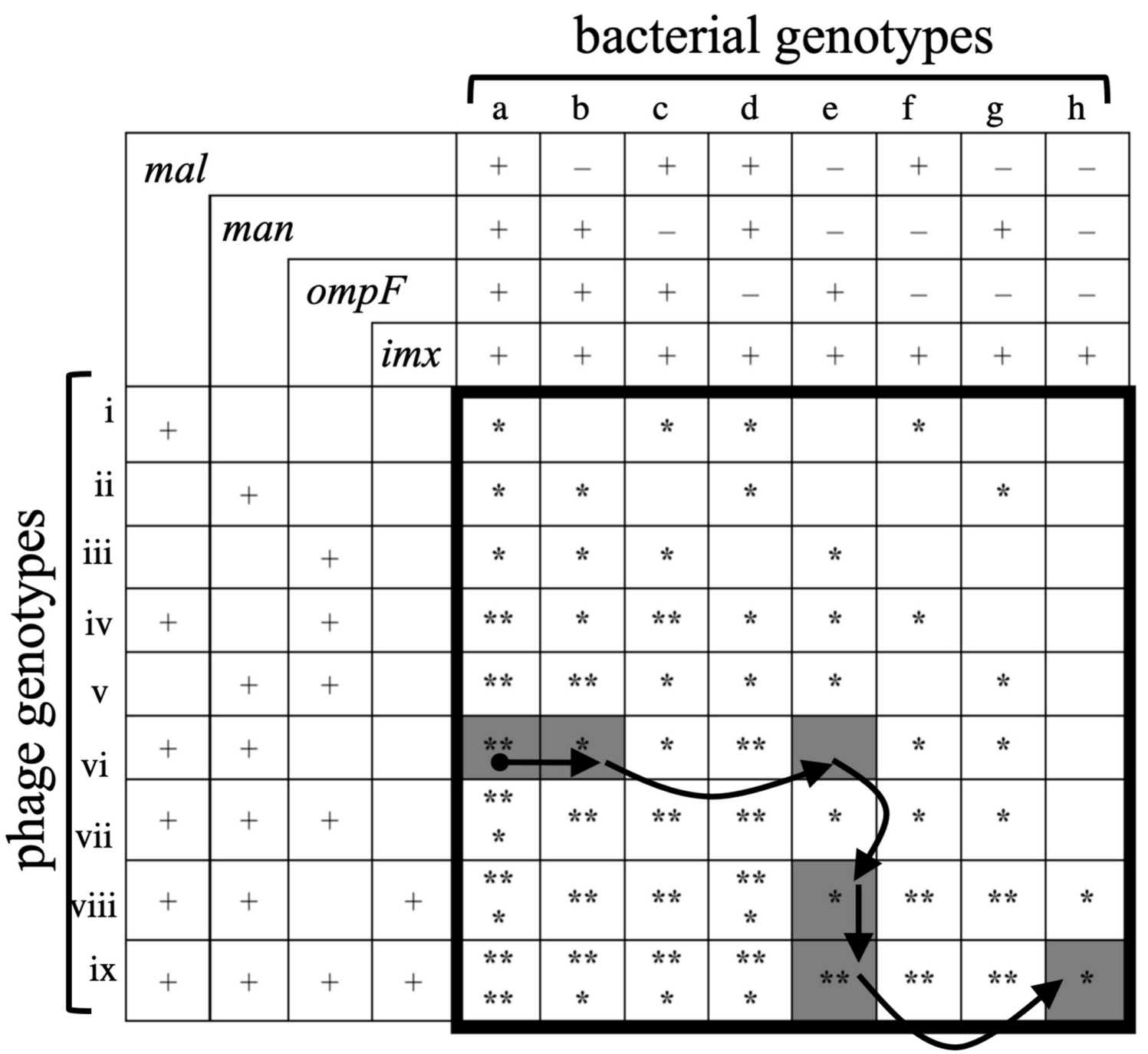

Fig. 5. An inverse gene-for-gene model showing the structure of the genetic network for coevolving $E$. coli and $\lambda$ populations. Columns indicate bacterial genotypes with four exploitable features, and rows indicate $\lambda$ genotypes that exploit those features: mal, maltose transport across the outer membrane; man, mannose transport across the inner membrane; omp $F$, glucose and electrolyte transport across the outer membrane; imx, a hypothetical inner membrane feature that is exploited by $\lambda$ that evolved independence of the mannose permease. The '+' symbol indicates that either the bacteria have the feature or the phage exploit the feature. The '-' symbol indicates that the bacteria lack the feature, express it to a reduced degree, or otherwise modify it to minimize phage infection. Asterisks $\left({ }^{*}\right)$ indicate infectivity for each host-phage pair, with more asterisks indicating greater infectivity. Adaptive changes through the network can proceed by two types of move: E. coli resistance (to the right along rows) and increased $\lambda$ infectivity (downward along columns). The coevolving communities were founded by host genotype a and phage genotype vi (shown by the black circle). The communities analysed in this study appear to have moved through the shaded nodes in five steps, as indicated by the arrows.

the bacteria's expression of LamB and thus the adsorption of the ancestral phage [33]; and (ii) $\lambda$ 's new ability to adsorb to $\mathrm{OmpF}$ as an alternative receptor [17]. Our phage growth data demonstrate that man $Y$ and manZ deletions confer substantial resistance even to the ancestral phage, which can use only the LamB surface receptor (Fig. 2). That result suggests the possibility that the man mutants could have arisen early in the coevolution experiments, perhaps alongside or even before the malT mutations that provided resistance at the outer membrane. However, time course data show that the man alleles consistently reached high frequencies (above the detection limits, shown as grey dashed lines in Fig. 3) only after the fixation of the malT mutations, which occurred by day 8 in both populations studied here (Figs. 3 and 4, Table S3) [17].

These temporal data also show that the man mutations had nearly fixed in both bacterial populations (frequencies $>95 \%$ on day 10 in Pop-A and on day 12 in Pop-B), but then the mutants sharply declined the next day. This reversal suggests that these mutants were killed by phages that evolved independence of the mannose permease, and it is consistent with previous data 
showing that mutant man alleles rarely fixed in the bacterial populations [17]. Meyer et al. (Fig. S2 in [17]) reported that the bacterial population densities remained high $\left(\sim 2 \times 10^{9}\right.$ cells $\mathrm{ml}^{-1}$, near the carrying capacity of the medium) throughout this period of the evolution experiment. Therefore, the mutant frequencies that we observed (Fig. 3) correspond to $\sim 4 \times 10^{7}$ cells $\mathrm{ml}^{-1}$ (about $2 \%$ of the total population, the limit of detection in that assay) to almost $2 \times 10^{9}$ cells $\mathrm{ml}^{-1}$ (the carrying capacity). With such large population sizes, any phage mutants that gained the ability to infect the man mutants would have access to a large number of hosts, and correspondingly, a large fitness benefit. The resulting growth of the man-independent phage population would drive the frequency of man mutants down, especially if the man-independent phages preferentially infected and killed the man mutants relative to other cells that retained the wildtype permease. Fitness costs associated with loss of the mannose permease may also have contributed to the reversal, although the costs of the resistance mutations are small compared to their benefit in the presence of phage [36].

In host Pop-A, variation in colony morphology further suggested that different man alleles were present before and after the sudden decline in the frequency of man mutants on day 11 (Fig. 4, Supplementary Results and Discussion). The initial boom and bust of the mutant man alleles in both populations also occurred before the phage had evolved to use OmpF (Fig. 3, dashed arrows). Whether $\lambda$ gained independence from the mannose permease by exploiting another inner-membrane protein, and whether E. coli did (or could) respond by eliminating such a structure, are interesting questions for future work.

Our results are broadly consistent with genetic and molecular biology studies of $\lambda$ host range mutations. Scandella and Arber [30] isolated E. coli mutants that allowed phage adsorption to the cell envelope but interfered with ejection of the phage genome, thereby reducing infection success to a small fraction of that observed on wild-type cells. The responsible mutations were mapped to the mannose permease operon $[27,37]$, and $\lambda$ mutants that could infect these mutant bacteria had mutations in phage genes $V$ or $H$ [38]. Mutations in $V$ and $H$ have also been observed in another population in this study system [39]. Williams et al. [37] found that, for E. coli strain $\mathrm{K} 12$, manZ is not strictly required for wild-type $\lambda$ to eject its genome, and our results are in accord with that finding (Fig. 2, Keio background). However, our results suggest that $\lambda$ cI 26 does require man $Z$ when infecting $E$. coli strain B, at least in the culture conditions that we used (Fig. 2, REL606 background). Alternatively, $\lambda$ cI26 might occasionally infect and replicate in hosts without manZ, but at a rate that is offset by the decay or inactivation of free virus particles under these conditions $[17,33]$. In any case, the net population growth of the ancestral phage on either the $\Delta m a n Y$ or $\triangle m a n Z$ bacteria is insufficient to offset the 100-fold daily dilutions (Fig. 2, dashed line) that took place during the coevolution experiment [17].

Taken together, our results imply that $E$. coli and $\lambda$ coevolved in an inverse gene-for-gene manner [18] (Fig. 1). This coevolution involved two infection steps
- crossing first the outer and then the inner membrane and at least three, and probably four, distinct host features (Figs 1,5$)$. E. coli evolved resistance to phage $\lambda$ through the loss or alteration of maltose transport across the outer membrane (via mutations in malT) and mannose transport across the inner membrane (via mutations in man $Y$ or $\operatorname{manZ}$ ), while $\lambda$ evolved to exploit other $E$. coli features, including another outer-membrane protein $(\mathrm{OmpF})$ and, presumably, some as yet unidentified, alternative inner-membrane protein (shown as encoded by the hypothetical imx gene in Fig. 5). While our study addresses one particular bacteria-phage interaction in a simple laboratory setting, it illustrates the extent to which the resulting coevolutionary arms races can be richer and more complex than is often appreciated.

There are many alternative coevolutionary paths through an inverse gene-for-gene network that has four features subject to host defences and parasite counter-defences (Fig. 5). This multiplicity of potential paths suggests that mutation and selection could drive replicate communities to different regions of the coevolutionary landscape, raising other interesting questions. How might different first-step resistance mutations affect the subsequent host range evolution of the phage and the further evolution of host resistance? To what extent can IGFG systems continuously evolve host defences and parasite counterdefences? What is the effect of such prolonged coevolution for community diversity? Do communities become increasingly divergent as the coevolving populations follow different paths through the network, or might they eventually converge on the same phenotypic states after a period of divergence? How important are evolutionary innovations in opening new paths, relative to pleiotropic tradeoffs that may close off certain paths? Future work should investigate these and other questions about the coevolution of bacteria and phage and the structure of their genetic interaction networks.

\footnotetext{
Funding information

This work was supported by the National Science Foundation Graduate Research Fellowship (DGE-1424871) to A. R. B., the BEACON Center for the Study of Evolution in Action (NSF Cooperative Agreement DBI-0939454), the John Hannah endowment from Michigan State University to R. E. L., a Marie Curie IIF Fellowship to J. G., and the Max Planck Society to J. G. The funders had no role in study design, data collection and interpretation, or the decision to submit the work for publication. Any opinions, findings, and conclusions or recommendations expressed in this paper are those of the authors and do not necessarily reflect the views of the funders.
}

\section{Acknowledgements}

We thank Neerja Hajela for assistance in the lab; Justin Meyer for advice on experimental methods, sharing samples from the coevolution experiments, and valuable discussions; and Rohan Maddamsetti, Caroline Turner, Mike Wiser, and Mike Blazanin for comments on the manuscript.

Author contributions

A. R. B., R. M. S. and R. E. L. conceived the study. A. R. B., R. M. S. and J. G. performed the experiments. All authors analysed the data and wrote the manuscript.

Conflicts of interest

The authors declare that there are no conflicts of interest. 
References

1. Lenski RE, Levin BR. Constraints on the coevolution of bacteria and virulent phage: a model, some experiments, and predictions for natural communities. Am Nat 1985;125:585-602.

2. Chao L, Levin BR, Stewart FM. A complex community in a simple habitat: an experimental study with bacteria and phage. Ecology 1977;58:369-378

3. Levin BR, Stewart FM, Chao L. Resource-limited growth, competition, and predation: a model and experimental studies with bacteria and bacteriophage. The American Naturalist 1977;111:3-24.

4. Waterbury JB, Valois FW. Resistance to co-occurring phages enables marine Synechococcus communities to coexist with cyanophages abundant in seawater. Appl Environ Microbiol 1993;59:3393-3399.

5. Williams Smith $\mathrm{H}$, Huggins MB. The association of the 018, K1 and $\mathrm{H} 7$ antigens and the ColV plasmid of a strain of Escherichia coli with its virulence and immunogenicity. Microbiology 1980;121:387-400.

6. Williams Smith $\mathrm{H}$, Huggins MB. Successful treatment of experimental Escherichia coli infections in mice using phage: its general superiority over antibiotics. J Gen Microbiol 1982;128:307-318.

7. Burmeister AR, Fortier A, Roush C, Lessing AJ, Bender RG, et al. Pleiotropy complicates a trade-off between phage resistance and antibiotic resistance. Proc Natl Acad Sci U S A 2020;117:11207-11216.

8. Chan BK, Sistrom M, Wertz JE, Kortright KE, Narayan D, et al. Phage selection restores antibiotic sensitivity in MDR Pseudomonas aeruginosa. Sci Rep 2016;6:26717

9. Kortright KE, Chan BK, Koff JL, Turner PE. Phage therapy: A renewed approach to combat antibiotic-resistant bacteria. Cell Host Microbe 2019;25:219-232.

10. Barrangou R, Fremaux C, Deveau H, Richards M, Boyaval P, et al. CRISPR provides acquired resistance against viruses in prokaryotes. Science 2007:315:1709.

11. Shmakov S, Smargon A, Scott D, Cox D, Pyzocha N, et al. Diversity and evolution of class 2 CRISPR-Cas systems. Nat Rev Microbiol 2017;15:169-182

12. Westra ER, Levin BR. It is unclear how important CRISPR-Cas systems are for protecting natural populations of bacteria against infections by mobile genetic elements. Proc Natl Acad Sci USA 2020;117:27777-27785

13. Pawluk A, Davidson AR, Maxwell KL. Anti-CRISPR: discovery, mechanism and function. Nat Rev Microbiol 2018;16:12

14. Buckling A, Rainey PB. Antagonistic coevolution between a bacterium and a bacteriophage. Proceedings of the Royal Society of London Series B. Proc Biol Sci 2002;269:931-936.

15. Scanlan PD, Buckling A. Co-evolution with lytic phage selects for the mucoid phenotype of Pseudomonas fluorescens SBW25. ISME J 2012;6:1148-1158.

16. Scanlan PD, Hall AR, Lopez-Pascua LDC, Buckling A. Genetic basis of infectivity evolution in a bacteriophage. Mol Ecol 2011;20:981-989.

17. Meyer JR, Dobias DT, Weitz JS, Barrick JE, Quick RT, et al. Repeatability and contingency in the evolution of a key innovation in phage lambda. Science 2012;335:428-432.

18. Fenton A, Antonovics J, Brockhurst MA. Inverse-gene-for-gene infection genetics and coevolutionary dynamics. Am Nat 2009;174:E230-242.

19. Fenton A, Antonovics J, Brockhurst MA. Two-step infection processes can lead to coevolution between functionally independent infection and resistance pathways. Evolution 2012;66:2030-2041.

20. Sieber M, Robb M, Forde SE, Gudelj I. Dispersal network structure and infection mechanism shape diversity in a coevolutionary bacteria-phage system. ISME J 2014;8:504-514.
21. Agrawal A, Lively CM. Infection genetics: gene-for-gene versus matching-alleles models and all points in between. Evol Ecol Res 2002:4:79-90

22. Dennehy JJ. What can phages tell us about host-pathogen coevolution? Int J Evol Biol 2012;2012:396165.

23. Koskella B, Brockhurst MA. Bacteria-phage coevolution as a driver of ecological and evolutionary processes in microbial communities. FEMS Microbiol Rev 2014;38:916-931.

24. Chaudhry WN, Pleška M, Shah NN, Weiss H, McCall IC, et al. Leaky resistance and the conditions for the existence of lytic bacteriophage. PLoS Biol 2018;16:e2005971.

25. Hofnung $M$, Jezierska A, Braun-Breton C. lamB mutations in E. coli K12: growth of lambda host range mutants and effect of nonsense suppressors. Mol Gen Genet 1976;145:207-213.

26. Thirion JP, Hofnung M. On some genetic aspects of phage lambda resistance in E. coli K12. Genetics 1972;71:207-216.

27. Elliott J, Arber W. E. coli K-12 pel mutants, which block phage lambda DNA injection, coincide with pts $M$, which determines a component of a sugar transport system. Mol Gen Genet 1978;161:1-8.

28. Erni B, Zanolari B, Kocher HP. The mannose permease of Escherichia coli consists of three different proteins: amino acid sequence and function in sugar transport, sugar phosphorylation, and penetration of phage lambda DNA. J Biol Chem 1987;262:5238-5247.

29. Esquinas-Rychen M, Erni B. Facilitation of bacteriophage lambda DNA injection by inner membrane proteins of the bacterial phosphoenol-pyruvate: carbohydrate phosphotransferase system (PTS). J Mol Microbiol Biotechnol 2001;3:361-370.

30. Scandella D, Arber W. An Escherichia coli mutant which inhibits the injection of phage lambda DNA. Virology 1974;58:504-513.

31. Spanakis E, Horne MT. Co-adaptation of Escherichia coli and coliphage $\lambda$ vir in continuous culture. J Gen Microbiol 1987;133:353-360.

32. Meyer JR, Agrawal AA, Quick RT, Dobias DT, Schneider D, et al. Parallel changes in host resistance to viral infection during 45,000 generations of relaxed selection. Evolution 2010;64:3024-3034

33. Burmeister AR, Lenski RE, Meyer JR. Host coevolution alters the adaptive landscape of a virus. Proc Roy Soc B: Biol Sci 2016;283:20161528.

34. Meyer JR, Flores CO, Weitz JS, Lenski RE. Key innovation in a virus catalyzes a coevolutionary arms race. ALife Proceedings 2008;13:532-533.

35. Baba T, Ara T, Hasegawa M, Takai Y, Okumura Y, et al. Construction of Escherichia coli K-12 in-frame, single-gene knockout mutants: the Keio collection. Mol Syst Biol 2006;2:0008.

36. Burmeister AR, Sullivan R, Lenski RE. Fitness costs and benefits of resistance to phage lambda in experimentally evolved Escherichia coli. Banzhaf W, Cheng B, Deb K, Holekamp K, Lenski RE, et al. (eds). In: Evolution in action: Past, present, and futuraction: Past, present, and future. New York, NY: Springer; 2020. pp. 123-143.

37. Williams N, Fox DK, Shea C, Roseman S. Pel, the protein that permits lambda DNA penetration of Escherichia coli, is encoded by a gene in pts $M$ and is required for mannose utilization by the phosphotransferase system. Proc Natl Acad Sci U S A 1986;83:8934-8938.

38. Scandella D, Arber W. Phage lambda-DNA injection into Escherichia coli pel- mutants is restored by mutations in phage gene $\mathrm{V}$ or gene H. Virology 1976;69:206-215.

39. Gupta A, Peng S, Leung CY, Borin JM, Weitz JS, et al. Leapfrog dynamics in phage-bacteria coevolution revealed by joint analysis of cross-infection phenotypes and whole genome sequencing. bioRxiv 2020.

Edited by: M. Brockhurst 